\title{
Rotation and Mass Ejection: the Launching of Be-Star Disks
}

\author{
Stanley P. Owocki \\ Bartol Research Institute, University of Delaware, Newark, DE 19350 \\ USA \\ Department of Physics and Astronomy, University of Glasgow, Glasgow \\ G12 $8 Q Q$ UK
}

\begin{abstract}
.
The characteristic signature of Be stars is the Balmer line emission understood to arise in a circumstellar disk. Unlike the accretion disks of protostars or mass-exchange binary systems, the evolved and generally single or wide-binary status of $\mathrm{Be}$ stars seems to require that its disk must form from mass ejection (a.k.a. decretion) from the star itself. In this paper, I use analogies with launching orbital satellites to discuss two candidate processes (radiation, pulsation) for driving such orbital mass ejection, with particular emphasis on the role of the rapid, possibly near-critical, rotation of Be stars in facilitating the formation of their signature disks.
\end{abstract}

\section{The Puzzle of Be Disks}

Within the context of this Symposium, an especially good laboratory to study the role of rapid stellar rotation is provided by the classical Be stars, which are among the most rapidly rotating massive stars. Be stars are main-sequence or subgiant spectral type B stars characterized by Balmer emission (e.g., $\mathrm{H} \alpha$ ) that originates in a circumstellar disk. Understanding the nature of such stars, and in particular the origin of their circumstellar disks, is one of the longest-standing challenges in astronomy (Secchi 1867; Slettebak \& Snow 1987; Balona, Henrichs \& LeContel 1994; Smith, Henrichs \& Fabregat 1999).

Disks are a common consequence in astrophysical accretion systems, wherein they form as a means to provide outward viscous transport of the angular momentum of the infalling material (Frank et al. 1992). Such accretion disks occur, for example, in protostellar nebulae, or in close binary systems with mass exchange. But Be stars are clearly too old to have retained a protostellar disk (indeed, in many Be stars the Balmer emission signatures of a disk are observed to come and go on timescales of months to decades), and moreover they are not generally found to be in close, mass-exchange binary systems. Thus lacking an outside source of material, it seems instead that Be disks must originate from ejection or decretion of mass from the underlying star. Identifying the specific dynamical mechanisms for achieving this "inside-out" formation of a circumstellar disks is perhaps the central puzzle underlying the Be phenomenon.

Dating back to early analyses by Struve (1931), rapid rotation has long been presumed to be a key piece in this puzzle. But as emphasized below ( $\S \S 3$, 
7), a crucial, still-open question is just how close Be star rotation might be to the "critical" speed, at which the equatorial surface would be in Keplerian orbit. Critical rotation would enable a direct centrifugal ejection of a circumstellar disk or ring, much as first envisioned by Struve, and developed further in modern "Viscous Decretion Disk" models of Lee et al. (1991) that are reviewed in these proceedings by A. Okazaki. But conventional interpretations of observed linebroadening are that Be star rotations are typically only $70-80 \%$ of critical, and in this case solving the puzzle of Be disk formation amounts to identifying the dynamical processes that provide the additional boost into circumstellar orbit.

One possibility is for mass ejection from localized surface explosions, e.g. perhaps from magnetic flares $(\S 4)$. The overview here briefly introduces some new dynamical simulations based on radiative driving (§5) and stellar pulsation (§6). [MHD simulations relevant to the more global Magnetically Torqued Disk (MTD) scenario promoted by Cassinelli et al. (2002) are discussed in a related talk write-up from the recent South Africa conference on stellar magnetic fields; see Owocki \& ud-Doula (2003) and also ud-Doula (2002)]. Then $§ 7$ describes recent reanalyses of the role of gravity darkening on inferred rotation rates, emphasizing in particular that these seem to reopen the possibility that Be rotation speeds could indeed be very close to the critical value. The conclusion ( $\S 8$ ) summarizes alternative scenarios for Be star rotation, emphasizing the implications and outlook for future dynamical modelling of Be disk formation, as well as for understanding the role of rapid rotation in massive stars.

\section{Lessons from the Wind Compressed Disk Paradigm}

In what seemed at first a very promising approach, the Wind Compressed Disk (WCD) model introduced by Bjorkman \& Cassinelli (1993) naturally produces an equatorial disk of enhanced-density flow from just rotational focussing of the radiatively driven stellar wind expected from the intrinsically bright $\mathrm{Be}$ stars. Initial dynamical simulations did in fact confirm much of the basic WCD paradigm (Owocki, Cranmer \& Blondin 1994), but subsequent work showed that non-radial (poleward) components of the driving can effectively inhibit the formation of any disk (Owocki, Cranmer \& Gayley 1996).

Moreover even within the initial WCD picture based on purely radial driving, the outflow from a subcritically rotating star necessarily lacks the angular momentum for a stationary orbit. As such, material in the inner disk is pulled by gravity into reaccretion back onto the star, while material in the outer disk flows outward with the stellar wind. This overall "leaking" of the WCD limits its density to values well below (by ca. a factor 100) that needed to explain either the observed Balmer emission or continuum polarization (Bjorkman 1999).

These substantial radial flow speeds also do not seem compatible with observed line-profile features, most notably the "central quasi-emissions" (Rivinius et al. 1999; Hanuschik 1995).

Finally, since this radially flowing WCD material has a characteristic residence time of only a few days, the WCD model seems inherently incapable of explaining the long-term (several year) variations of Violet/Red $(V / R)$ emission peak asymmetries seen in many Be stars. Instead these seem consistent with slow one-arm disk-oscillation modes that are grounded in the gradual precession 
of elliptical orbits within a Keplerian disk. (Savonije \& Heemskerk 1993; Telting et al. 1994; Savonije 1998).

Although it thus now seems quite clear that the WCD mechanism can not explain Be star emission, its introduction and development was nonetheless a quite useful and instructive step forward, as one of the first dynamically based models with falsifiable predictions. In particular, it highlighted that among key requirements for producing a viable disk are not just to propel material from the surface and focus it toward the equator, but rather also to provide it with sufficient angular momentum to maintain a Keplerian orbit

\section{Subcritical Equatorial Rotation and the Orbital Launch Speed}

Before discussing specific driving mechanisms, it is helpful to review the general requirements for propelling material into orbit from a rotating surface. As reflected in the choice of launch sites for terrestrial satellites, this is easiest when the launch occurs from near the equator and into the direction of rotation. Specifically, for a body with equatorial rotation speed $V_{\text {eq }}$ that is less than the orbital (a.k.a. "critical rotation") speed $V_{\text {crit }} \equiv \sqrt{G M / R_{\text {eq }}} \approx 500 \mathrm{~km} \mathrm{~s}^{-1}$, a prograde equatorial launch requires an additional speed boost of $\Delta V_{\text {orb }} \equiv V_{\text {crit }}-V_{\text {eq }}$.

Compared to launch from a nonrotating surface (or into a polar orbit), the energy required is reduced by a factor $\left(\Delta V_{\text {orb }} / V_{\text {crit }}\right)^{2}$. For earth satellites, this energy saving is modest but non-negligible - about $10 \%$. But for a typical massive star (e.g. Zeta Puppis) that rotates at half the critical speed, the required energy is reduced by a much more substantial factor four. And for Be stars, the traditional estimates of rotation rates up to $80 \%$ of critical imply an impressive factor 25 reduction in the energy to reach orbit!

Despite these significant reductions, the boost needed to reach orbit can in some respects still be quite large. For example, an alternative figure of merit is to compare the launch speed to the atmospheric sound speed, which for Be stars with surface temperatures of $T \approx 20,000 \mathrm{~K}$ has a typical value $a \approx 20 \mathrm{~km} \mathrm{~s}^{-1}$. Then even for a star rotating at $80 \%$ of the critical speed $V_{\text {crit }} \approx 500 \mathrm{~km} \mathrm{~s}^{-1}$, one finds a ratio $\Delta V_{\text {orb }} / a \approx 5$. This moreover implies that the internal, thermal energy of gas in Be star atmospheres would still fall short - in this case by roughly a factor $\left(\Delta V_{\text {orb }} / a\right)^{2} \approx 25$ - the minimal energy to accelerate material into orbit.

This last perspective suggests that mechanisms linked to the surface internal energy - in particular nonradial pulsation - are only likely to be effective for orbital ejection if rotation rates are quite close to critical, i.e. within one or two sound speeds. Before describing simulations of such pulsational mass ejection at near-critical rotation, let us first consider a radiatively driven ejection model that in principal can be effective even for more subcritical rotation.

\section{Mass Ejection by Surface Explosions or Magnetic Flares}

The minimum launch speed defined above requires in general that the launched material be properly directed along the sense of rotation. But an interesting alternative, first examined through Smoothed Particle Hydrodynamics (SPH) 
simulations by Kroll (1995; see also Kroll \& Hanuschik 1997), is to consider the effect of an undirected, explosive ejection of material from a localized equatorial region of a rapidly rotating surface. Despite the lack of prograde directivity, Kroll's SPH results show the natural formation of an equatorial disk through a kind of "Keplerian natural selection", in which material that happens to be propelled in the direction of rotation gains sufficient velocity to achieve circumstellar orbit, while material ejected in other directions simply falls back in a reaccretion onto the stellar surface.

In Kroll's simulations, the exploding material is arbitrarily given an initial velocity $\Delta V \approx 100-200 \mathrm{~km} \mathrm{~s}^{-1}$ that is sufficient to achieve orbit, with no consideration for what specific driving mechanism could propel the gas to this speed. Driving by gas pressure from a surface flare would require a comparable sound speed $(a \approx \Delta V)$, implying gas temperatures of order $10^{6} \mathrm{~K}$, much higher than what characterizes the observed optical emission.

Perhaps driving without too much heating could occur directly via magnetic pressure, which then requires a comparable value for the Alfven speed, $V_{A} \equiv$ $B / \sqrt{4 \pi \rho} \approx \Delta V$, with $\rho$ the density. Using mass continuity for an average mass ejection rate of $\dot{M}=4 \pi R_{*}^{2} \rho \Delta V f$, where $f$ is the product of areal and temporal 'filling factors' for the ejections, the required localized magnetic field scales as

$$
B=\frac{\sqrt{\dot{M} \Delta V / f}}{R_{*}} .
$$

For example, to build a disk against disspative losses that should be comparable to the stellar wind mass loss, let us take $\dot{M}=10^{-9} M_{\odot} /$ year. Then using a typical B-star radius $R_{*}=5 R_{\odot}$, we find explosions to $\Delta V=100 \mathrm{~km} \mathrm{~s}^{-1}$ with a $1 \%$ filling factor $(\mathrm{f}=0.01)$ require a localized field of just $B \approx 20 \mathrm{G}$.

If we replace $\Delta V / f$ with a stellar wind terminal speed $V_{\infty}$, then Eq. (1) also gives the minimal global field necessary to channel a wind mass loss of rate $\dot{M}$ (ud-Doula \& Owocki 2002). A slightly higher field moreover suffices to confine the flow into closed loops that corotate with the star, with strong-field loops extending up to where the corotation meets or exceeds the local speed for Keplerian orbit (ud-Doula 2002). However, as discussed in Owocki \& ud-Doula (2003), so far detailed MHD simulations show that wind material trapped and centrifugally held against the tops of such magnetic loops tends to periodically break out as radial mass ejections, rather than forming the "Magnetically Torqued Disk" postulated in the recent scaling analysis by Cassinelli et al. (2002).

\section{Radiatively Driven Orbital Mass Ejection}

As another alternative, I have recently been examining models in which driving is provided by the radiative force from localized bright spots on the stellar surface, a scenario I dub "Radiatively Driven Orbital Mass Ejection" (RDOME). A key feature of these RDOME models is to include the prograde radiative force that is expected in the region ahead of the bright spot. Since radiative driving is routinely capable of driving a stellar wind to speeds well in excess of orbital launch speeds, it seems in principal possible that the prograde force ahead of 
a.

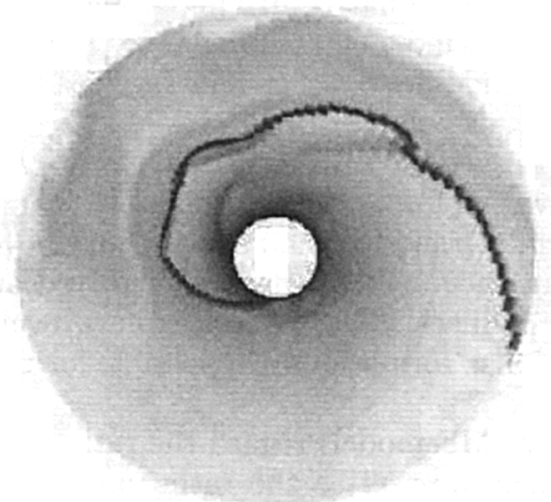

b.

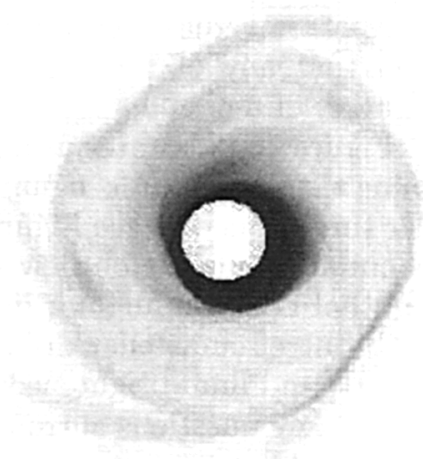

Figure 1. a. Density at fixed time snapshot of 2D, equatorial-plane models of flow radiatively driven from a bright spot on a star rotating at $70 \%$ critical, showing formation of dense CIR. b. Same as (a), but with a radiative force cutoff for $r>1.25 R_{*}$, resulting in fall-back of material into an orbiting disk. The central white circle corresponds to the star's equatorial diameter.

the bright spot might impart material there with sufficient angular momentum to achieve circumstellar orbit.

For a star with equatorial rotation speed $\left(350 \mathrm{~km} \mathrm{~s}^{-1}\right)$ that is $70 \%$ of the critical speed $\left(500 \mathrm{~km} \mathrm{~s}^{-1}\right)$, Figure 1a shows a typical time snapshot of the density from $2 \mathrm{D}$ radiation hydrodynamical simulations of the outflow driven by a spot with a localized brightness that peaks at a (quite large) factor of 10 times the ambient brightness. Confined to a region with Gaussian half-width of 10 degrees in longitude, this spot is suddenly turned on at some initial time within an otherwise steady-state wind model, and then shut off again after just $100 \mathrm{ksec}$, about a rotation period. Note that, instead of forming an orbiting circumstellar disk, the overall result is to propel a spiral Co-rotating Interaction Region (CIR) of enhanced density within the outflowing stellar wind. This is much the same form thought to be needed to explain Discrete Absorption Components in UV wind lines (Owocki, Cranmer \& Fullerton 1995; Cranmer \& Owocki 1996). The reason to favor wind CIRs over disks is that material initially propelled ahead of the spot tends eventually (due to its extension away from the surface) to fall behind the solid body angular rotation, and so as it thus comes over the central bright spot it receives a very strong radial push from the bright-spot radiative force, thus propelling it away from the star in an outflowing wind CIR.

There are a variety of possibilities for instead favoring lateral over radial driving from a bright spot. One is to consider a model in which, perhaps due to distortion of the surface associated with stellar pulsation, the spot radiation might have prograde-bias in the direction of rotation. Another is to assume a symmetric, but highly limb-brightened spot emission, with the upward intensity comparatively suppressed, somewhat analogous to the emission pattern from 
a quiescent solar prominence, which appears bright above the solar limb, but relatively dark against the solar disk. A third possibility (first suggested by J. Bjorkman, p.c.) is to assume a cutoff in the radiative driving above some relatively low radius, perhaps reflecting shifts in wind ionization balance. Indeed, I find that any of these rather specialized modifications can in fact lead to formation of a circumstellar disk.

Figure 1b illustrates a snapshot of the disk density for the last scenario, assuming the same spot as figure $1 \mathrm{a}$, but now with a force cutoff beyond $1.25 R_{*}$. A fuller time sequence (see Figure 5 of Owocki \& Cranmer 2002) shows that above this cutoff radius the outflow stagnates, but since material has also received an azimuthal boost in angular momentum, its fallback toward the star now forms an orbiting circumstellar disk. I find similar disk formations are possible for the prograde and limb-brightened spot scenarios.

The key questions surrounding such RDOME models regard the rather extreme brightness variations that are assumed, as well as the rather arbitrary fine-tuning of the spot emission or line-force cutoff. Brightness fluctuations are sometimes observed in Be stars, but further work is needed to determine whether these have a time-scale and magnitude that might be consistent with this RDOME scenario.

Indeed, recent analyses suggest that, quite apart from propelling material to feed a circumstellar disk, radiative forces may actually play a key role is dissipating an existing disk, for example through "line-driven ablation", which could drive a wind outflow from the disk surface (Gayley et al. 1999, 2001; Rivinius et al. 2001). From this perspective, radiative forces might instead be considered an "enemy of the disk", perhaps of more relevance for their intermittent disappearance whenever other mechanisms for ejecting fresh material into orbit are inoperative or reduced.

\section{Pulsationally Driven Orbital Mass Ejection}

Recent observations by Rivinius et al. (1998, 1999) suggest that in at least one Be star ( $\mu$ Cen) there may be a coincidence between multiperiodic beating of nonradial pulsation (NRP) modes and outbursts in circumstellar lines that signify star-to-disk mass transfer.

Inspired by these observations, H. Mueller and I have recently been carrying out $2 \mathrm{D}$ hydrodynamical simulations of the effectiveness of equatorial pulsations in inducing orbital mass ejections. For the considered periods ( $\sim 0.5$ day) well above the acoustic-cutoff period, the pulsations are taken to be gravity modes, with the restoring force due to buoyancy transmitted through the gas pressure. Since this is primarily a circulation rather than a compression, the velocity amplitude could feasibly approach or even slightly exceed the sound speed, with the motions primarily in the lateral vs. radial direction. In our simulations, this azimuthal velocity variation is introduced as an Eulerian perturbation at the star's equatorial surface, with corresponding logarithmic variation in density (typically factor 10) to account for the associated vertical oscillation within an exponentially stratified atmosphere. A positive (negative) phase relation between azimuthal velocity and density then corresponds to an NRP with phase propagation that is prograde (retrograde) to the sense of rotation. 


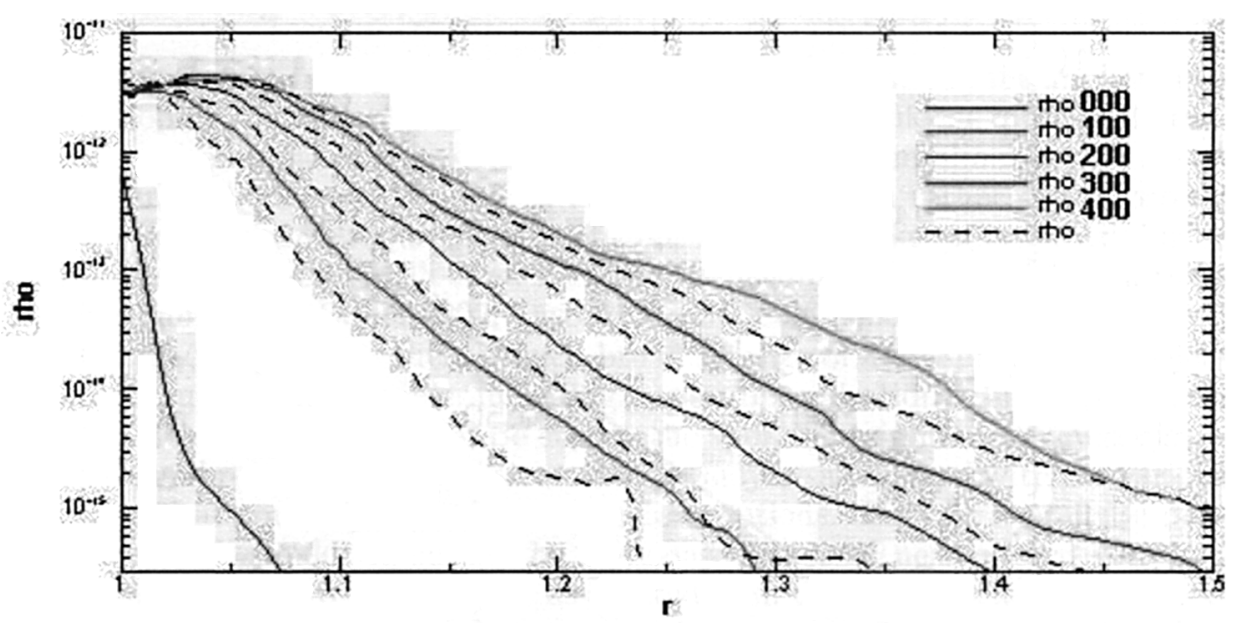

Figure 2. Azimuthally averaged density vs. radius at fixed intervals of 50 ksec after turnon of non-radial pulsation in a star with rotation of $95 \%$ critical. The resulting ejection of material forms an orbiting disk whose density increases with time.

In accord with the orbital launch requirements outlined in $\S 3$, we generally find that substantial disk production requires a prograde pulsation with an azimuthal velocity amplitude $\Delta V_{\mathrm{NRP}}$ that is comparable to the orbital launch speed $\Delta V_{\text {orb. }}$. As a typical example, Figure 2 illustrates results for an $\mathrm{m}=+4$ prograde NRP with azimuthal velocity amplitude $\Delta V_{\mathrm{NRP}}=25 \mathrm{~km} \mathrm{~s}^{-1}$ that is equal to the required launch speed $\Delta V_{\text {orb }}$, corresponding to an equatorial rotation speed $V_{\mathrm{eq}} \approx 0.95 V_{\text {crit }}$. The need for prograde modes stands in apparent contradiction with the interpretation by Rivinius et al. (1999) that NRPs observed in $\mu$ Cen correspond to retrograde modes. This, and the need to assume near-critical rotation, thus represent key issues for the viability of this scenario for "Pulsationally Driven Orbital Mass Ejection" (PDOME).

\section{Equatorial Gravity Darkening and Saturation of Line-Broadening}

As reviewed by G. Collins \& I. Howarth in these proceedings, the principal diagnostic for Be star rotation stems from the rotational broadening of photospheric absorption lines, most commonly He I $\lambda 4471$. The observed half-widths of such lines are typically characterized by a velocity-unit measure called Vsini, which for low to moderate rotations is directly proportional to a star's equatorial rotation velocity $V_{\mathrm{eq}}$ times the inclination factor $\sin i$, with $i$ the angle of the rotation axis to the observer.

However, for rapid rotation within about $20-30 \%$ of the critical speed, the Von Zeipel (1924) gravity darkening of equatorial regions significantly lowers their relative contribution to the overall line-broadening. Using the BRUCE NLTE line synthesis code (Townsend 1997) applied to rotationally distorted 


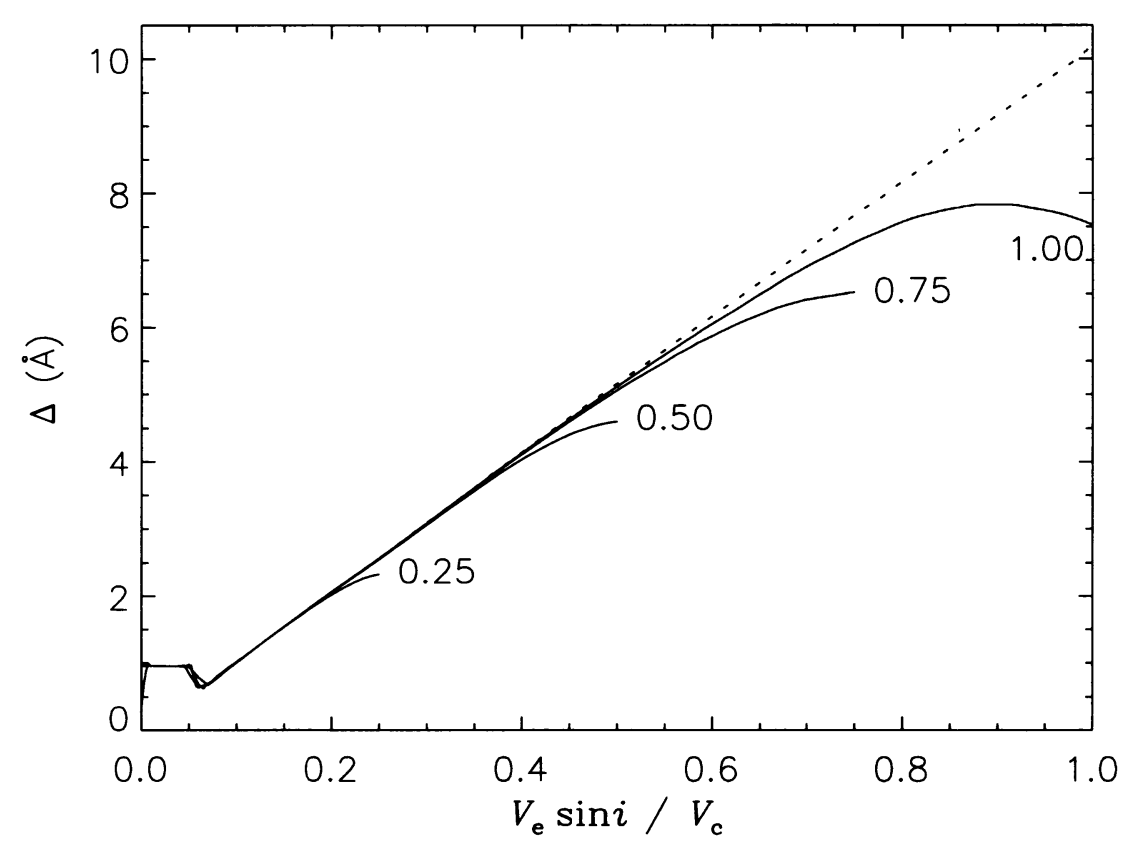

Figure 3. Expected broadening of He I $\lambda 4471$ plotted against the projected equatorial speed $V_{\text {eq }} \sin i$ scaled by the critical speed $V_{\text {crit }}$. The labels of the various curves denote the inclination factor $\sin i$.

and gravity darkened B2 stars of various assumed inclinations, Figure 3 plots the expected width of He I $\lambda 4471$ against the actual $V_{\mathrm{eq}} \sin i$, scaled in units of the critical speed $V_{\text {crit }}$. Note particularly that for stars with $V_{\text {eq }} \sin i / V_{\text {crit }} \gtrsim 0.7$, the measured Vsini falls below a linear relation, indeed becoming essentially independent of the actual rotation for $V_{\text {eq }} \sin i / V_{\text {crit }} \gtrsim 0.8$.

Results similar to Figure 3 were actually obtained long ago by Stoeckley (1968; cf. his Figure 4, and also, e.g., Figure 6 of Collins \& Truax 1995), and indeed led him to speculate explicitly that measured line-broadenings might actually be consistent with the general population Be stars being at essentially critical rotation. Rather inexplicably, however, in the modern era the potential ramifications of gravity darkening in this context do not appear to have been fully appreciated or explored, even though typical inclination-corrected rotation rates for Be stars are quoted to be at levels approaching $80 \%$ critical, at which Figure 3 shows an effective saturation of measured line broadening. Thus even within current-day measurement errors and analysis techniques, it still seems quite plausible that the typical rotation speeds might actually be significantly higher than commonly quoted, perhaps even within a few percent of critical rotation! Clarification of these issues should be a top priority for Be-star research. 


\section{Concluding Outlook}

If Be-star rotation rates are indeed found to be very near critical, the implications for developing dynamical models of Be disk formation would be quite profound. As emphasized in $\S 3$, a key parameter is the ratio of the minimal launch speed to the atmospheric sound speed. If $\Delta V_{\text {orb }} / a \gg 1$, then a rather strong orbital ejection mechanism is required, with obvious candidates like radiative driving or magnetic spin-up (Cassinelli et al. 2002) so far needing rather special conditions and fine-tuning (ud-Doula 2002; Owocki \& ud-Doula 2003).

But if $\Delta V_{\text {orb }} / a \sim 1$, then a much broader range of atmospheric-based ejection mechanisms are possible, including non-radial pulsations. In this case, the complex behavior of individual Be stars - the sporadic brightenings, even disappearance and reappearance of disk emission - may be associated with the various processes that could spark individual mass eruptions. But the overall Be phenomenon itself would essentially be the consequence of near-critical rotation, owing perhaps to envelope spin-up associated with the evolutionary contraction of a massive-star's core toward the terminal age main sequence, as reviewed in these proceedings by G. Meynet \& A. Maeder.

From this perspective, the disk emission that characterizes Be stars could be considered the result of interior-driven processes that require equatorial shedding of excess angular momentum, somewhat like a rotational analog of the Rochelobe overflow in mass-exchange binary systems. In both cases, a disk forms as a repository for the ejected material, with its net mass - and thus its observed emission strength - depending on the competition between the stellar ejection rate and the rate for disk dissipation, e.g. from radial diffusion and perhaps surface ablation. Although the overall mass ejection is controlled by interior processes, the details can be variable and spatially complex, controlled by surface disturbances. Indeed the dynamics and energetics of the specific perturbation processes can even influence how much below the critical level the mean surface remains.

To conclude, I believe a top priority for Be-star research should be the development of dynamical models of disk production within a context of more firmly established observational constraints on how close the stellar rotation may be to critical. Within a broader astrophysical context of this conference, I also suggest that, instead of being held as rather isolated oddities, Be stars should be explored as potentially important general laboratories for studying the causes and consequences of evolutionary trends toward critical rotation in massive stars.

Acknowledgments. This paper was written while visiting University of Glasgow under support of a UK-PPARC Visitor Fellowship, and I am grateful to J. Brown and others in Glasgow for their hospitality. The research herein was also supported in part by NASA grant NAG5-3530 and NSF grant AST-0097983 to the University of Delaware. I thank H. Mueller for Figure 2 and R. Townsend for Figure 3. I also thank D. Baade and S. Stefl for helpful comments on an initial draft. 


\section{References}

Bjorkman, J. E. \& Cassinelli, J. P. 1993, ApJ 409, 429.

Bjorkman J. E., 1999, Variable and Nonspherical Stellar Winds in Luminous Hot Stars, B. Wolf, O. Stahl \& A. Fullerton (eds.). IAUC 169, Lecture Notes in Physics 523, Springer, p. 121

Cassinelli, J. P., Brown, J. C., Maheswaran, M., Miller, N. A. \& Telfer, D. C. 2002, ApJ 578,951

Collins, G. W. \& Truax, R. J. 1995, ApJ 439, 860

Cranmer, S. R. \& Owocki, S. P. 1996, ApJ 462, 469

Frank, J., King, A. \& Raine, D. 2002, Accretion Power in Astrophysics, Cambridge University Press: Cambridge, UK

Gayley, K. G., Owocki, S. P. \& Cranmer, S. R. 1999, ApJ 513, 442

Gayley, K. G., Ignace, R. \& Owocki, S. P. 2001, ApJ 558, 802

Hanushik, R. W. 1995, A\&A 295, 423

Kroll, P. 1995, Ph. D. Thesis, U. of Tuebingen, Tuebingen, Germany

Kroll P. \& Hanuschik R.W., 1997, Accretion Phenomena \& Related Outflows, IAUC 163, Wickramasinghe D.T., et al. (eds.), PASPC 121, p. 494

Lee, U. \& Saio, H. \& Osaki, Y. 1991, MNRAS 250, 432

Maeder, A. \& Meynet, G. 2001, A\&A 372, L9

Owocki, S. P., Cranmer, S. R. \& Blondin, J. 1994, ApJ 424, 887

Owocki, S. P., Cranmer, S. R. \& Fullerton, A. W.. 1995, ApJ 453, 370

Owocki, S. P., Cranmer, S. R. \& Gayley, K. G. 1996, ApJ 472, L115

Owocki, S. P. \& Cranmer, S. R. 2002, in "Radial and Nonradial Pulsations as Probes of Stellar Physics", IAU Colloquim 185, C. Aerts, T. Beddings \& J. ChristensenDalsgaard, eds., ASP Conference Proceedings, vol. 259, p. 512

Owocki, S. P. \& ud-Doula, A. 2003, in "Magnetic Fields in O, B, and A Stars", T. Medupe \& L. Balona, eds., in preparation

Rivinius, T. J. 1998, Ph.D. Thesis, U. Heidelberg

Rivinius, T., Stefl, S. \& Baade, D. 1999, A\&A 348, 831

Rivinius, T., Baade, D., Stefl, S. \& Maintz, M. 2001, A\&A 379, 257

Savonije, G. J. 1998, in Cyclical Variability in Stellar Winds, L. Kapers \& A. Fullerton, eds., Springer: Berlin, p. 337

Savonije, G. J. \& Heemskerk, M. H. M. 1993, A\&A 276, 409

Secchi, A. 1867, Astron. Nach. 68, 63

Slettebak A. \& Snow, T. 1987, Physics of Be Stars, Proceedings of IAUC 96, Cambridge University Press

Smith, M. A., Henrichs, H. H. \& Fabregat, J. 2000, The Be Phenomenon in Early-Type Stars, IAU Colloquium 175, ASP Conference Proceedings, Vol. 214

Stoeckley, T. R. 1968, MNRAS 140, 141

Struve, O. 1931, ApJ 73, 94

Telting, J. H., Heemskerk, M. H. M., Henrichs, H. F., \& Savonije, G. J. 1994, A\&A 288, 558

Townsend, R. H. D. 1997, Ph. D. Thesis, University College London

ud-Doula, A. 2002, Ph. D. Thesis, University of Delaware

ud-Doula, A. \& Owocki, S. P. 2002, ApJ 576, 413

von Zeipel, H. 1924, MNRAS 84, 665 